\title{
The claustrum is required for reward acquisition under high cognitive demand
}

$4{ }^{1}$ Department of Pharmacology, University of Maryland School of Medicine, Baltimore,

5 MD, USA. ${ }^{2}$ Allen Institute for Brain Sciences, Seattle, WA, USA

Acknowledgments: This work was supported by National Institute on Alcohol Abuse and Alcoholism grants K22AA021414 and R01AA024845 (B.N.M.), Whitehall Foundation grant 2014-12-68 (B.N.M.), National Institute of General Medical Sciences grant T32GM008181 (M.G.W.), National Institute of Neurological Disorders and Stroke grant T32NS063391 (M.G.W.), and National Institute of Mental Health grant F31MH112350

12 (M.G.W.). The authors also are grateful for the assistance of Dr. Christof Koch and the Allen Institute for Brain Science.

15 TThese authors contributed equally to this work

$16{ }^{*}$ Correspondence should be addressed to:

17 Brian N. Mathur

18 BRB 4-011

19655 West Baltimore Street

20 Baltimore, MD 21201

21 Tel: (410) 706-8239

\section{BMathur@som.umaryland.edu}




\section{Abstract}

The claustrum is proposed to mediate a variety of functions ranging from sensory

27 binding to top-down cognitive control of action, but direct functional assessments of this

28 telencephalic nucleus are lacking. Here we employ the guanine nucleotide-binding

29 subunit beta-4 cre driver line in mice to selectively monitor and manipulate claustrum

30 projection neurons. Using fiber photometry, we find elevated claustrum activity prior to

31 an expected cue during correct performance on a cognitively demanding five-choice

32 response assay relative to a less-demanding one-choice version of the task. Claustrum

33 activity during reward acquisition is also enhanced when cognitive demand is higher.

34 Furthermore, we use optogenetic inhibition of claustrum prior to the expected cue to

35 demonstrate that claustrum is critical for accurate performance on the five-choice, but

36 not the one-choice, task. These results suggest the claustrum supports a cognitive

37 control function necessary for reward acquisition under cognitively demanding conditions. 


\section{Introduction}

Across species, the claustrum is widely connected with the neocortex including sensory, motor, association and executive cortices (Crick and Koch 2005; Mathur 2014). This connectivity motivates a number of functional hypotheses (Remedios et al. 2010; Smith and Alloway 2010; Smythies et al. 2012; Mathur 2014; Patru and Reser 2015), including that the claustrum binds sensory information to generate conscious percepts (Crick and Koch 2005). Direct analysis of claustrum function is historically intractable, which necessitated indirect functional assessments of this structure. For instance, a recent study leveraged the dense anterior cingulate cortex input to claustrum (Smith and Alloway 2010; Wang et al. 2017; White et al. 2017) to show that activity of this circuit rises with, and is required for, optimal performance on a five-choice response task (White et al. 2018).

Novel transgenic tools, such as cre recombinase driver lines, now provide genetic access to the claustrum (Wang et al. 2017). In this study, we use the guanine nucleotide binding protein beta 4 cre driver line (GNB4-cre) for monitoring and manipulation of claustrum projection neurons in awake, freely moving mice. Given the role of the anterior cingulate cortex input to the claustrum on five-choice response task performance, we herein examine the role of the claustrum itself on this task.

Understanding claustrum function stands to inform higher order brain functions involving functional coordination across the cortical mantle (Miller and Buschman 2013; Koch et al. 2016; Tononi et al. 2016; White and Mathur 2018a). 


\section{Results}

72

73

74

75

76

77

78

79

80

81

82

\section{Claustrum contributes to complex, but not simple, task performance}

To confirm that claustrum projection neurons express cre recombinase in the GNB4-cre mouse, we injected a virus expressing eYFP in a cre-dependent manner into the claustrum of GNB4-cre mice (Figure 1A). We used parvalbumin (PV) immunostaining to delineate claustrum borders (Figure 1B) (Mathur et al. 2009; White et al. 2018) and observed that virus expression and PV immunostaining were isomorphic (Figure 1C). We next examined the morphological and electrophysiological identity of GNB4-positive (+) neurons by performing whole-cell recordings from labeled neurons using recording pipettes filled with AlexaFluor®-594. We found that GNB4+ neurons were spiny (Figure 1D), consistent with a projection neuron identity (Braak and Braak 1982; Hur and Zaborszky 2005; Watakabe et al. 2014). Basic membrane properties of these neurons are shown in Figure 1E and representative responses to current injection steps are shown in Figure 1F. Because capacitance delineates two claustrum projection neurons (White and Mathur 2018b), this membrane property can further identify GNB4+ neurons. In particular, we find a wide range of GNB4+ capacitance values (89 to 176 pF) consistent with sampling from both subtypes (type I = $118 \pm 16 \mathrm{pF}$; type II = $158 \pm 9$ pF [mean \pm SD]; White and Mathur 2018b).

To determine if claustrum is critical for the five-choice serial reaction time task (5CSRTT) performance, we injected cre-dependent halorhodopsin (AAV-DIOeNPhR3.0) or eYFP (AAV-DIO-eYFP) into the claustrum of GNB4-cre mice and implanted optical fibers to expose the claustrum to $470 \mathrm{~nm}$ light (Figure 2A). In acute brain slices, $470 \mathrm{~nm}$ light readily blocked action potential generation in claustrum 
neurons expressing eNPhR3.0 during a depolarizing voltage step (Figure 2B).

Experimental and control mice were trained to perform the 5CSRTT and subsequently a one-choice stimulus-response task (1CSRTT; Figure 2C). The 1CSRTT was used to control for the basic sensory and motor components of the 5CSRTT. The claustrum was exposed to $470 \mathrm{~nm}$ light during the inter-trial interval (ITI) pseudo-randomly on 33\% of 5CSRTT and 1CSRTT trials (Figure 2D). This protocol was derived from our previous work showing ACC input to claustrum is critical for optimal task performance within $1 \mathrm{~s}$ of cue onset (White et al. 2018). On 5CSRTT trials paired with $470 \mathrm{~nm}$ stimulation, AAV-DIO-eNPhR3.0 mice were less accurate compared to control trials; whereas no difference in accuracy was observed in AAV-DIO-eYFP mice (Figure 2E). Light delivery did not change the number of omissions or the response latency on correctly performed 5CSRTT trials in AAV-DIO-eNPhR3.0 or AAV-DIO-eYFP mice (Figure 2F and 2G). For AAV-DIO-eNPhR3.0 mice, we found that accuracy deficits on inactivation trials during the 1CSRTT were less than those on the 5CSRTT (Figure 2H). For AAV-DIO-eYFP mice, no differences in accuracy deficits were observed between the two tasks (Figure $2 \mathrm{H})$.

To further exclude possible motor or reward-related effects elicited by claustrum inactivation, we performed a real-time place preference (RTPP) assay. We found that both AAV-DIO-eNPhR3.0 and AAV-DIO-eYFP mice did not exhibit a preference for either the control side of the chamber or the side of the chamber paired with $470 \mathrm{~nm}$ light stimulation (Figure 2 - Supplement 1A and 1B). Assessing whether claustrum inactivation affects movement, we found no difference in the velocity of AAV-DIO- 
eNPhR3.0 and AAV-DIO-eYFP mice before $470 \mathrm{~nm}$ light exposure compared to the $1 \mathrm{~s}$ of inactivation + the $1.5 \mathrm{~s}$ following inactivation (Figure 2 - Supplement $1 \mathrm{C}$ ).

\section{Claustrum activity is sensitive to task load}

We used a custom-made in vivo fiber photometry system to monitor calciumdependent activity of claustrum in GBN4-cre mice injected with a virus expressing GCaMP6f in a cre-dependent manner (AAV-FLEX-GCaMP6f; Figure 3A). The system and data processing were designed to minimize sources of noise, such as fluctuations in excitation laser intensity, motion-related artifacts, and bleaching artifacts. Fluctuations in excitation laser intensity were controlled for by exciting a stable fluorophore, AlexaFluor ${ }^{\circledR}-488(\mathrm{AF} \AA-488$; Figure 3 - Supplement 1A-1C). Motion-related artifacts were controlled for by multiplexing excitation of claustrum with $405 \mathrm{~nm}$ light, the isosbestic wavelength for GCaMP6 (Kim et al. 2016), and $473 \mathrm{~nm}$ light (Figure Supplement $1 \mathrm{~A}$ and $1 \mathrm{~B})$. Regressing out the signals from $405 \mathrm{~nm}$ excitation and $\mathrm{AF} \circledast$ 488 excitation attenuated noise (Figure 3 - Supplement 1D-1F). To control for photobleaching during 30 min 5CSRTT sessions, signals were examined in 10 trial bins.

During 5CSRTT performance, claustrum activity was elevated on correct and omission trials in the early phase of the ITI relative to incorrect trials (Figure 3B). In the late phase of the ITI, there were no differences in claustrum activity among the different trial types (Figure 3B). We next aligned claustrum activity to correct and incorrect nose pokes during 5CSRTT performance. Average claustrum activity around the time of nose poke was significantly greater for correct nose pokes compared to incorrect nose pokes (Figure 3C). To assess if claustrum activity bears any relationship with movement, we 
139 monitored activity during free movement in an open field (Figure 3D). Claustrum activity was weakly and negatively correlated with movement velocity (Average $r=-0.0062$

141 Figure 3E). We next examined if claustrum activity during correct 5CSRTT performance

142 reflects task load. To this end, we first compared claustrum activity during the ITI

143 between correctly performed 5CSRTT and 1CSRTT trials (Figure 4A) and found

144 relatively enhanced claustrum activity during the early ITI on the 5CSRTT but no

145 differences during the late ITI (Figure 4B). We next compared the two tasks by aligning

146 activity to the correct nose pokes (Figure 4C). We did not observe any activity

147 differences between the two tasks immediately prior to or subsequent to nose pokes

148 (Figure 4D). Lastly, we aligned claustrum activity to the acquisition of sucrose pellets

149 (Figure 4E). Activity was greater for the 5CSRTT relative to the 1CSRTT immediately 150 before and after acquisition of the sucrose pellet (Figure 4F).

152 Discussion

154 cognitively-demanding 5CSRTT but not the 1CSRTT or open field movement.

155 Claustrum activity on the 5CSRTT is higher on accurate compared to inaccurate task

156 performance. In addition, relative to the 1CSRTT, claustrum activity on the 5CSRTT is

157 higher at early stages prior to the cue and at reward acquisition. These findings are in

158 line with the previous finding that the activity of anterior cingulate cortex inputs to

159 claustrum are greater during 5CSRTT than 1CSRTT (White et al. 2018) and support the 160 notion that the claustrum is involved in cognitive control of action. 
It is important to note that our photometry analysis detects population level

162

163

164

165

166

167

168

claustrum projection neuron activity. Therefore, it is difficult to determine, for example, whether increased activity-dependent calcium signals reflect recruitment of more claustrum neurons, an increase in firing of a subset of claustrum neurons, synchrony of claustrum neurons, or some combination of these possibilities. As such, future studies will need to assess if selective activation of functionally distinct subpopulations may explain our finding that claustrum activity on omission trials, as with correct trials, is elevated relative to incorrect trials during the 5CSRTT ITI, for example.

Claustrum activity may also be driven by retrosplenial cortex, which is heavily connected with claustrum (White et al. 2017), encodes disengagement from the external environment in humans (Buckner et al. 2008) and is part of a homologous task negative network in rodent (Lu et al. 2012). Given the claustrum's widespread cortical interconnectivity and response to top-down command (White et al. 2018), the present results suggest a role for the claustrum in supporting cortical networks underlying cognitive control of goal-directed behavior under demanding conditions.

\section{References}

Braak H, Braak E. 1982. Neuronal types in the claustrum of man. Anat Embryol (Berl). 163:447-460.

Buckner RL, Andrews-Hanna JR, Schacter DL. 2008. The Brain's Default Network: Anatomy, Function, and Relevance to Disease. Ann N Y Acad Sci. 1124:1-38.

Crick FC, Koch C. 2005. What is the function of the claustrum? Philos Trans R Soc B 
Biol Sci. 360:1271-1279.

184

185

186

187

188

189

190

191

192

193

194

195

196

197

198

199

200

201

202

203

Dalley JW, Cardinal RN, Robbins TW. 2004. Prefrontal executive and cognitive functions in rodents: neural and neurochemical substrates. Neurosci Biobehav Rev. 28:771-784.

Hur EE, Zaborszky L. 2005. Vglut2 afferents to the medial prefrontal and primary somatosensory cortices: a combined retrograde tracing in situ hybridization study [corrected]. J Comp Neurol. 483:351-373.

Kim CK, Yang SJ, Pichamoorthy N, Young NP, Kauvar I, Jennings JH, Lerner TN, Berndt A, Lee SY, Ramakrishnan C, Davidson TJ, Inoue M, Bito H, Deisseroth K. 2016. Simultaneous fast measurement of circuit dynamics at multiple sites across the mammalian brain. Nat Methods. 13:325-328.

Koch C, Massimini M, Boly M, Tononi G. 2016. Neural correlates of consciousness: progress and problems. Nat Rev Neurosci. 17:307-321.

Lu H, Zou Q, Gu H, Raichle ME, Stein EA, Yang Y. 2012. Rat brains also have a default mode network. Proc Natl Acad Sci U S A. 109:3979-3984.

Mathur BN. 2014. The claustrum in review. Front Syst Neurosci. 8:48.

Mathur BN, Caprioli RM, Deutch AY. 2009. Proteomic analysis illuminates a novel structural definition of the claustrum and insula. Cereb Cortex. 19:2372-2379.

Miller EK, Buschman TJ. 2013. Cortical circuits for the control of attention. Curr Opin Neurobiol. 23:216-222.

Muir JL, Everitt BJ, Robbins TW. 1996. The Cerebral Cortex of the Rat and Visual 
Attentional Function: Dissociable Effects of Mediofrontal, Cingulate, Anterior Dorsolateral, and Parietal Cortex Lesions on a Five-Choice Serial Reaction Time Task. Cereb Cortex. 6:470-481.

Passetti F, Chudasama Y, Robbins TW. 2002. The Frontal Cortex of the Rat and Visual Attentional Performance: Dissociable Functions of Distinct Medial Prefrontal Subregions. Cereb Cortex. 12:1254-1268.

Patru MC, Reser DH. 2015. A New Perspective on Delusional States - Evidence for Claustrum Involvement. Front psychiatry. 6:158.

Remedios R, Logothetis NK, Kayser C. 2010. Unimodal Responses Prevail within the Multisensory Claustrum. J Neurosci. 30:12902-12907.

Robinson ESJ, Eagle DM, Mar AC, Bari A, Banerjee G, Jiang X, Dalley JW, Robbins TW. 2008. Similar Effects of the Selective Noradrenaline Reuptake Inhibitor Atomoxetine on Three Distinct Forms of Impulsivity in the Rat. Neuropsychopharmacology. 33:1028-1037.

Smith JB, Alloway KD. 2010. Functional Specificity of Claustrum Connections in the Rat: Interhemispheric Communication between Specific Parts of Motor Cortex. J Neurosci. 30:16832-16844.

Smythies J, Edelstein L, Ramachandran V. 2012. Hypotheses relating to the function of the claustrum. Front Integr Neurosci. 6:53.

Tononi G, Boly M, Massimini M, Koch C. 2016. Integrated information theory: from consciousness to its physical substrate. Nat Rev Neurosci. 17:450-461. 
225 Wang Q, Ng L, Harris JA, Feng D, Li Y, Royall JJ, Oh SW, Bernard A, Sunkin SM, Koch

226

227

228

229

230

231

232

233

234

235

236

237

238

239

240

241

242

243

244

245

C, Zeng H. 2017. Organization of the connections between claustrum and cortex in the mouse. J Comp Neurol. 525:1317-1346.

Watakabe A, Ohsawa S, Ichinohe N, Rockland KS, Yamamori T. 2014. Characterization of claustral neurons by comparative gene expression profiling and dye-injection analyses. Front Syst Neurosci. 8:98.

White MG, Cody PA, Bubser M, Wang H-D, Deutch AY, Mathur BN. 2017. Cortical hierarchy governs rat claustrocortical circuit organization. J Comp Neurol. $525: 1347-1362$.

White MG, Mathur BN. 2018a. Frontal cortical control of posterior sensory and association cortices through the claustrum. Brain Struct Funct. 1-8.

White MG, Mathur BN. 2018b. Claustrum circuit components for top-down input processing and cortical broadcast. Brain Struct Funct. Accepted.

White MG, Panicker M, Mu C, Carter AM, Roberts BM, Dharmasri PA, Mathur BN. 2018. Anterior Cingulate Cortex Input to the Claustrum Is Required for Top-Down Action Control. Cell Rep. 22:84-95.

\section{Materials and methods}

Animals. 34 GNB4-cre mice bred from a C57BL/6J background of both sexes were used (Wang et al. 2017). Mice used for electrophysiology were 10-20 weeks of age at the time of experiments and group-housed with food and water available ad libitum. In 
contrast, mice used for behavioral experiments were 16-30 weeks of age at the time of experiments. These mice were singly-housed, weighed daily, and fed daily to maintain $90 \%$ of ad libitum weigh. All mice were on a $12 \mathrm{~h}$ light-dark cycle beginning at 0700 and 5CSRTT experiments were performed during the light cycle. For optogenetic experiments, control and experimental groups were comprised of mice from the same litters to minimize any litter effects. This study was performed in accordance with the National Institutes of Health Guide for Care and Use of Laboratory Animals and the University of Maryland, School of Medicine, Animal Care and Use Committee.

\section{Viral vectors and stereotaxic procedures.}

For optogenetic inhibition or in vivo fiber photometry monitoring of claustrum 80$110 \mathrm{~nL}$ of $\mathrm{AAV}$ vectors expressing loxP-flanked double inverted open reading frames (DIO) of halorhodopsin (AAV5-eF1a-DIO-eNPhR3.0-eYFP; University of Pennsylvania Vector Core) or GCaMP6f (AAV9-EF1a-DIO-GCaMP6f; University of Pennsylvania Vector Core), respectively, were injected bilaterally at two rostrocaudal levels of the claustrum (4 injections) in GNB4-cre mice. In control mice and mice used for whole-cell electrophysiology, the same approach was used but with a vector expressing eYFP (AAV5-eF1a-DIO-eYFP; University of Pennsylvania Vector Core). Relative to bregma, claustrum coordinates were 1) anterior-posterior: $+1.34 \mathrm{~mm}$, medial-lateral $\pm 2.3 \mathrm{~mm}$, dorsal-ventral (from the brain surface): $-2.35 \mathrm{~mm}$; and 2) anterior-posterior: $+0.86 \mathrm{~mm}$, medial-lateral $\pm 2.75 \mathrm{~mm}$, dorsal-ventral (from the brain surface): $-2.55 \mathrm{~mm}$. Viral incubation was no fewer than 3 weeks. 
272 Mice used for in vivo fiber photometry received a unilateral implant into claustrum, which

273 was custom-made from low (0.22) NA fiber and ceramic ferrules (ThorLabs Inc; Newton,

274 NJ). Fiber implant placement was confirmed post-hoc using immunohistochemistry. The

275 light path angle $(\theta)$ was approximated to ensure accurate implantation using the known

Ex vivo brain slice preparation for electrophysiology. Following anesthetization, mice were decapitated, the brains were extracted, and $250 \mu \mathrm{m}$ coronal sections were sliced using a vibrating microtome in a high-sucrose artificial cerebrospinal fluid (aCSF). The aCSF was ice-cold, carbogen $\left(95 \% \mathrm{O}_{2}, 5 \% \mathrm{CO}_{2}\right)$-bubbled, and consisted of $194 \mathrm{mM}$ sucrose, $30 \mathrm{mM} \mathrm{NaCl}, 4.5 \mathrm{mM} \mathrm{KCl}, 1 \mathrm{mM} \mathrm{MgCl}_{2}, 26 \mathrm{mM} \mathrm{NaHCO}_{3}, 1.2 \mathrm{mM} \mathrm{NaH}_{2} \mathrm{PO}_{4}$,

286 and $10 \mathrm{mM}$ D-glucose. Sections were incubated after slicing for $30 \mathrm{~min}$ at $33^{\circ} \mathrm{C}$ in 287 carbogen-bubbled aCSF (315-320 mOsm) that contained $124 \mathrm{mM} \mathrm{NaCl}, 4.5 \mathrm{mM} \mathrm{KCl,} 2$ $288 \mathrm{mM} \mathrm{CaCl}_{2}, 1 \mathrm{mM} \mathrm{MgCl}_{2}, 26 \mathrm{mM} \mathrm{NaHCO}_{3}, 1.2 \mathrm{mM} \mathrm{NaH}_{2} \mathrm{PO}_{4}$, and $10 \mathrm{mM}$ D-glucose. 
290 recordings, and recordings were performed in the same aCSF formulation used for

291 incubation.

Whole-cell current and voltage-clamp recordings. Whole-cell recordings were performed

294 at $29^{\circ} \mathrm{C}-31^{\circ} \mathrm{C}$ using borosilicate glass recording pipettes of 3-7 $\mathrm{M} \Omega$ resistance.

295 Recording pipettes were filled with a potassium-based solution (290-295 mOsm; $\mathrm{pH} 7.3$ )

296 composed of $126 \mathrm{mM}$ potassium gluconate, $4 \mathrm{mM} \mathrm{KCl,} 10 \mathrm{mM}$ HEPES, 4 mM ATP-Mg,

$297 \quad 0.3$ mM GTP-Na and 10 mM phosphocreatine. Clampex software (version 10.4;

298 Molecular Devices; Sunnyvale, CA) was used for electrophysiological recordings, which 299 were filtered at $2 \mathrm{kHz}$ and digitized at $10 \mathrm{kHz}$. Internal pipette solutions also contained

300 hydrazide dye conjugated with AlexaFluor ${ }^{\circledR}-488(40 \mu \mathrm{M})$ for visualization of dendritic

301 spines. GNB4+ neurons expressing eYFP or halorhodopsin were identified using

302 epifluorescence and targeted for recordings. For neurons expressing halorhodopsin,

$303470 \mathrm{~nm}$ light was delivered with an external LED for $300 \mathrm{~ms}$ during a $1 \mathrm{~s}$ current

304 injection that elicited neuron firing to determine successful inhibition of firing using 470

$305 \mathrm{~nm}$ light.

307 Five-choice serial reaction-time task (5CSRTT). Mice were trained to perform the

308 5CSRTT (Muir et al. 1996; Passetti et al. 2002; Dalley et al. 2004; Robinson et al. 2008)

309 in operant chambers (Med Associates; St. Albans, VT) housed within sound-attenuating

310 cabinets as previously described (White et al. 2018). Briefly, mice are trained to nose

311 poke into one of five pseudo-randomly illuminated apertures (cue). Trials are preceded 
312 by a $5 \mathrm{~s}$ inter-trial interval (ITI) and responses are allowed during the cue and up to $5 \mathrm{~s}$

313 after the cue. Correct nose pokes resulted in a sucrose pellet dispensed into a

314 receptacle on the wall opposite to the five apertures. A new trial ITI did not begin until 5

315 s after the reinforcement was collected. Nose pokes into the incorrect aperture, no

316 response (omission), and nose pokes during the ITI resulted in a $5 \mathrm{~s}$ time out period,

317 during which the house light was extinguished. Any nose poke during the time out

318 period restarted the $5 \mathrm{~s}$ time out.

For optogenetic experiments, $470 \mathrm{~nm}$ light was delivered bilaterally during experimental sessions using an LED system (Plexon Inc; Dallas, TX); light delivery

321 occurred pseudo-randomly on 33\% of trials during the ITI period continuously for $1 \mathrm{~s}$

322 prior to the onset of the cue. A session ended after 100 trials or $30 \mathrm{~min}$, whichever occurred first, and data was averaged across five sessions. described (White et al., 2018). Mice were habituated to the operant chamber for two 15 min sessions with sucrose pellets available in the receptacle. Shaping occurred in two

327 phases. First, all apertures were illuminated and any nose poke was reinforced. Apertures were initially loaded with sucrose pellets to facilitate initial poking. In the second phase, only one aperture was illuminated and only a nose poke into the

330 illuminated aperture was reinforced. Mice progressed through each shaping phase after 331 meeting criterion, which was defined as 30 correct nose pokes in a 30 min session. No 332 time outs were given during shaping. 
cue duration was $10 \mathrm{~s}$ and for the second training phase, the cue duration was $5 \mathrm{~s}$. For training phases, criterion was defined as $60 \%$ accuracy and 30 responses within a session. Mice were over-trained on the final task ( $1 \mathrm{~s}$ cue) until choice accuracy reached a stable baseline before manipulations. Subsequent to 5CSRTT experimental sessions, mice were trained to perform a one-choice modification of the 5CSRTT (1CSRTT). All aspects of the task remained the same, except the middle aperture was illuminated and active on every trial. Mice were over-trained until performance stabilized before beginning experimental sessions. In 1CSRTT experimental sessions, $470 \mathrm{~nm}$ light was delivered as described above for the 5CSRTT continuously for $1 \mathrm{~s}$ prior to the onset of the cue.

\section{Real-time place preference (RTPP) and open field assay. The RTPP apparatus} consisted of a two-sided chamber connected with a narrow corridor. The RTPP assay consisted of habituation and test sessions. In the habituation session, mice were placed initially in the narrow corridor of the chamber and allowed free exploration for 20 min. On the following day, mice performed the test session, which paired one side of the chamber with $1 \mathrm{~s}$ of continuous $470 \mathrm{~nm}$ light bouts. These bouts were repeated at most every $20 \mathrm{~s}$ in order to approximate the amount of stimulation that occurred during the 5CSRTT sessions. The amount of time spent in each side of the chamber during sessions and ambulatory velocity was recorded with EthoVision XT v 11.5 (Noldus, Wageningen, The Netherlands). The velocity during the $0.5 \mathrm{~s}$ of light delivery and the subsequent $2 \mathrm{~s}$ was compared to the $2.5 \mathrm{~s}$ preceding light delivery to determine any effect of light delivery on movement. The same apparatus was used as an open field to 
358 measure calcium-dependent activity of claustrum during movement using in vivo fiber

359 photometry.

361 In vivo fiber photometry. Photometry data from 5CSRTT and open field experiments

362 were collected using a customized in vivo fiber photometry system. Two single

363 wavelength laser modules were used (Opto Engine LLC; Midvale, Utah), a $473 \mathrm{~nm}$ laser

364 for optimal GCamP6f excitation, and a $405 \mathrm{~nm}$ laser to excite GCaMP6f at its isosbestic 365 wavelength (Kim et al. 2016). As such, emission from $405 \mathrm{~nm}$ excitation was used to 366 control for image artifacts due to photometry cable motion, background fluorescence, 367 and other sources of noise (Kim et al. 2016). The two lasers were multiplexed at $10 \mathrm{~Hz}$, 368 resulting in a continuous $20 \mathrm{~Hz}$ pulse train. Both laser beams were directed using the 369 two-bounce method into a dichroic filter cube optimized for 473 and $405 \mathrm{~nm}$ excitation, 370 as well as for $510 \mathrm{~nm}$ emission (Chroma Technology Corporation; Bellow Falls, VT).

371 The two excitation wavelengths were focused through a $4 \mathrm{X}$ fluorite objective (Olympus;

372 Tokyo, Japan) onto a multimode fiber bundle that consisted of seven individual

373 multimode fibers. One fiber was connected to the mouse via chronic multimode fiber 374 implant, while another fiber was placed inside a tube of AlexaFluor®-488 $(40 \mu \mathrm{M})$ to 375 control for variability in laser energy. Emissions from GCaMP6f and AlexaFluorß-488

376 through the multimode fibers were detected as an image of the fiber bundle using the

377 ORCA Flash 4.0LT high-resolution CMOS camera (Hamamatsu Photonics KK;

378 Hamamatsu City, Japan). Laser multiplexing and image acquisition were synchronized

379 using an Arduino Leonardo microcontroller and time-locked to 5CSRTT trials when 
necessary. Camera image acquisition parameters were controlled through the HCImage Software for Hamamatsu cameras.

Data analysis and statistics. 5CSRTT photometry data were analyzed using a combination of MATLAB (Mathworks; Natick, Massachusetts) and Prism v 6.0.1 (GraphPad Software; La Jolla, CA). MATLAB Graphical User Interface (GUI) codes were written specifically to process the photometry raw image datasets, each one consisting of up to 40,000 images. First, the raw signal from the photometry signal fiber and the fluorophore control fiber was extracted by selecting ROls for both fibers, and then the pixel values were averaged across the entire ROI to extract the raw signal from the image. The raw signals were then subtracted from a corresponding background signal from the same image, which is the baseline transmission of ambient light from a photometry fiber when excitation lasers are off. The processed signals were then sorted based on if the image was taken during a 473 or $405 \mathrm{~nm}$ laser pulse. Further processing required a separate MATLAB GUI for linear regression and averaging each individual dataset across all trials. First, 473 and $405 \mathrm{~nm}$ photometry signals were regressed with the corresponding control fluorophore signal, and the residuals of the regression were then used for further processing. The $473 \mathrm{~nm}$ signal was then regressed with the 405 $\mathrm{nm}$ signal as a covariate, and the residuals of the regression were extracted as the fully processed photometry signal from the claustrum. The processed signal was then sorted based on trial type (correct, incorrect or omission), and averaged across trials for each specific trial type, and then across all five 5CRSTT runs for each animal. Further 
402

403

404

405

406

407

408

409

410

411

412

413

414

415

416

417

418

419

420

421

422

423

statistical analysis was performed in GraphPAD Prism. All statistics are displayed as mean \pm standard error unless otherwise noted.

\section{Figure legends}

Figure 1: The GNB4-cre transgenic mouse line provides genetic access to

claustrum projection neurons. (A) Cre-dependent viral expression of eYFP in mouse claustrum. (B) Immunostaining of claustrum parvalbumin (PV) expression. (C) Labeling of virally-expressed eYFP and immunolabeled PV were isomorphic. (D) Dendritic spines were evident upon whole-cell patch clamp records of GNB4-positive claustrum neurons using an internal solution including AlexFluor®-594. (E) Left: Mean capacitance from labeled neurons was $127 \pm 9$ (mean \pm SD) pF. Middle: Mean membrane resistance was $222 \pm 34 \mathrm{M} \Omega$. Right: Mean resting membrane potential was $-60 \pm 2 \mathrm{mV}$. (F)

Representative traces from labeled neuron in responsive to current injection steps. Horizontal scale bars $=200 \mu \mathrm{m}(\mathrm{A}-\mathrm{C}), 10 \mu \mathrm{m}(\mathrm{D}), 400 \mathrm{~ms}(\mathrm{~F})$. Vertical scale bars $=30$ $\mathrm{mV}$ (F [top]), $200 \mathrm{pA}$ (F [bottom]).

Figure 2: Inactivation of claustrum prior to cue presentation selectively disrupts response accuracy on the five-choice serial reaction time task (5CSRTT). (A) Left: Schematic illustrating that a virus expressing either halorhodopsin $(n=9)$ or eYFP $(n=$ 6) in a cre-dependent manner (AAV-DIO-eNPhR3.0 or -eYFP, respectively) was injected bilaterally into the claustrum (CL) of GNB4-cre mice. Optical fibers were 
424 chronically implanted bilaterally in the claustrum. Right: Photomicrograph illustrating

425 fiber optic implant (white box) position above claustrum, estimated light path (dotted

426 lines), and viral expression. (B) Representative trace from a claustrum neuron

427 expressing halorhodopsin (eNPhR3.0) that shows loss of action potential firing in the

428 presence of $470 \mathrm{~nm}$ light during a depolarizing current injection. (C) Mice were trained

429 to perform the 5CSRTT and subsequently a one-choice control task (1CSRTT). (D)

430 Experimental schematic illustrating that $470 \mathrm{~nm}$ light was delivered to the claustrum

431 during the inter-trial interval (ITI) 1 s prior to the onset of the cue on $33 \%$ of 5 CSRTT or

432 1CSRTT trials. (E) Top: For eNPhR3.0 mice performing the 5CSRTT, choice accuracy

433 was reduced on trials paired with $470 \mathrm{~nm}$ light delivery pre-cue compared to control

434 trials. Paired $t$ test, $t(8)=3.20, P=0.013$. Bottom: No changes in choice accuracy were

435 found on 5CSRTT trials paired with light delivery in eYFP mice. Paired $t$ test, $t(5)=1.38$,

$436 P=0.23$. (F) Top: The omission rate was not different on 5CSRTT trials paired with 470

$437 \mathrm{~nm}$ light delivery compared to control trials for eNPhR3.0 mice. Paired $t$ test, $t(8)=0.14$,

$438 P=0.91$. Bottom: Light delivery did not alter omission rate in eYFP mice. Paired $t$ test,

$439 t(5)=0.97, P=0.38$. (G) Top: The latency to correctly respond was not different on

440 5CSRTT trials paired with $470 \mathrm{~nm}$ light delivery compared to control trials for eNPhR3.0

441 mice. Paired $t$ test, $t(8)=1.24, P=0.25$. Bottom: Light delivery did not alter latency to

442 correctly respond in eYFP mice. Paired $t$ test, $t(5)=0.042, P=0.97$. $(\mathrm{H})$ Top: For

443 eNPhR3.0 mice, the reduction in choice accuracy on the 5CSRTT resulting from 470

$444 \mathrm{~nm}$ light delivery was significantly larger than the reduction during 1CSRTT

445 performance. Paired $t$ test, $t(8)=4.44, P=0.0022$. Bottom: For eYFP mice, there was

446 no difference in the change in choice accuracy on the 5CSRTT resulting from $470 \mathrm{~nm}$ 
447 light delivery compared to the change during 1CSRTT performance. Paired $t$ test, $t(5)=$

$4480.14, P=0.89$. Horizontal scale bars $=200 \mu \mathrm{m}(\mathrm{A}), 500 \mathrm{~ms}(\mathrm{~B})$. Vertical scale bar $=30$

$449 \mathrm{mV}$.

451 Figure 2 - Supplement 1: Claustrum inactivation is not reinforcing and does not

452 affect movement. (A) Top: Representative heat map from a GNB4-cre mouse

453 expressing eNPhR3.0 in claustrum during a real-time place preference assay (RTPP).

454 One side of the chamber was paired with 1 s $470 \mathrm{~nm}$ light delivery to claustrum that

455 cycled at a maximum rate of once every $20 \mathrm{~s}$ and the other side was not paired with

456 light. Hot colors indicate more time spent in a given location and cool colors indicate

457 less time spent in a given location. Bottom: Representative heat map from a GNB4-cre

458 mouse expressing eYFP in claustrum during the RTPP assay. (B) Top: For eNPhR3.0

459 mice, no difference was observed in the time spent on the side paired with $470 \mathrm{~nm}$ light

460 compared to the control side. Paired $t$ test, $t(8)=0.089, P=0.93$. Bottom: For eYFP

461 mice, no difference was observed in the time spent on the side paired with $470 \mathrm{~nm}$ light

462 delivery to claustrum compared to the control side. Paired $t$ test, $t(5)=1.20, P=0.28$.

463 (C) Top: Movement velocity during the $2.5 \mathrm{~s}$ interval prior to $470 \mathrm{~nm}$ light delivery was

464 compared to velocity during the $1 \mathrm{~s}$ of light delivery plus the $1.5 \mathrm{~s}$ interval following the

465 offset of light delivery. For eNPhR3.0 mice, no differences in velocity were observed

466 between the two intervals. Paired $t$ test, $t(8)=0.55, P=0.60$. Bottom: For eYFP mice,

467 no differences in velocity were observed between the two intervals. Paired $t$ test, $t(5)=$

$468 \quad 0.81, P=0.45$ 
470

471

472

473

474

475

476

477

478

479

480

481

482

483

484

485

486

487

488

489

490

491

492

Figure 3: Claustrum activity during 5CSRTT performance and open field behavior.

(A) Experimental schematic illustrating that claustrum activity was monitored during 5CSRTT performance in GNB4-cre mice injected with AAV-FLEX-GCaMP6f $(n=12)$.

(B) Left: Average calcium-dependent activity of claustrum aligned to 5CSRTT cue onset is shown for correct (green), incorrect (red), and omission (gray) trials. Middle: Early in the ITI (5 to 2.5 s prior to cue onset), claustrum activity was greater for correct and omission trials relative to incorrect trials. Repeated measures ANOVA, $F(2,11)=9.48, P$ $=0.0014$; post-hoc Tukey's test, $P=0.015$ (correct vs. incorrect), $P=0.0095$ (omission vs. incorrect). Right: Late in the ITI (2.5 to 0 s prior to cue onset), claustrum activity did not differ across trial types. Repeated measures ANOVA, $F(2,11)=1.975, P=0.17$. (C) Left: Average claustrum activity aligned to correct or incorrect nose pokes during 5CSRTT performance is shown. Right: Average claustrum activity (1 s prior to $1 \mathrm{~s}$ after poke) was greater for correct nose pokes compared to incorrect nose pokes. Paired $t$ test, $t(11)=3.14, P=0.0095$. (D) Left: Cartoon of mouse in an open field. Right: Representative traces of normalized claustrum activity (green) and velocity (black) during movement in an open field from GNB4-cre mice. (E) Cross-correlation between the photometry signal and movement velocity.

\section{Figure 3 - Supplement 1: Custom in vivo fiber photometry system and data}

processing. (A) Schematic illustrating the custom in vivo fiber photometry system used for monitoring claustrum activity. Two excitation lasers, $473 \mathrm{~nm}$ (odd numbered signals) and $405 \mathrm{~nm}$ (even numbered signals), were multiplexed at $10 \mathrm{~Hz}$. The beams were aligned, directed into a dichroic filter cube, and passed through a $4 \mathrm{X}$ objective into a 
493 multi-mode fiber bundle. One fiber was coupled to a chronic fiber implanted in the

494 claustrum (signals numbered 1 and 2) of GNB4-cre mice injected with a cre-dependent

495 virus expressing GCaMP6f (AAV-FLEX-GCaMP6f; inset). A control fiber was placed

496 inside a tube of AlexaFluor®-488 (AF-488) fluorophore (signals numbered 3 and 4).

497 GCaMP6f and AF-488 emissions collected by the fiber bundle were imaged with a

498 Hamamatsu CMOS camera. The camera captured images at $20 \mathrm{~Hz}$ synchronized to

499 each laser pulse. A fiber uncoupled to a fluorescence source (signals numbered 5 and

500 6) was used to measure background signals and obtain $\Delta F$ values. (B) Top:

501 Representative image showing the fiber bundle in response to $473 \mathrm{~nm}$ excitation.

502 Bottom: Representative image showing the fiber bundle in response to $405 \mathrm{~nm}$

503 excitation. (C) Top: Representative trace illustrating AF-488 $\Delta \mathrm{F}$ values in response to

$504473 \mathrm{~nm}$ excitation $(3-5)$. Bottom: Representative trace illustrating AF-488 $\Delta \mathrm{F}$ values in

505 response to $405 \mathrm{~nm}$ excitation (4 - 6). (D) Representative traces illustrating GCaMP6f

$506 \Delta \mathrm{F}$ values measured from claustrum with $473 \mathrm{~nm}$ excitation $(1-5)$ from a bin of 10

507 5CSRTT trials that include different trial types. (E) Representative traces illustrating

508 autofluorescence $\Delta F$ values measured from claustrum with $405 \mathrm{~nm}$ excitation $(2-6)$.

509 Arrow indicates example of a noise signal. (F) Representative traces illustrating

510 denoised GCaMP6f signals obtained following regression of the AF-488 signal (C, top)

511 and autofluorescence signal (E). Arrows indicate an attenuated noise signal and a true

512 signal.

514 Figure 4: Claustrum activity on 5CSRTT versus 1CSRTT. (A) Left: Average

515 claustrum activity during the early ITI for correct 5CSRTT trials (green) and correct 
1CSRTT trials (blue). Right: Average activity during the late ITI for correct 5CSRTT and

517 1CSRTT trials. (B) Left: Claustrum activity during the early ITI was greater for correct

518 5CSRTT trials compared to 1 CSRTT trials. Paired $t$ test, $t(11)=2.92, P=0.014$. Right:

519 no difference was observed between correct 5CSRTT and 1CSRTT trials during the late

520 ITI. Paired test, $t(11)=0.87, P=0.41$. (C) Left: Average claustrum activity aligned to

521 correct nose pokes during 5CSRTT performance and right: during 1CSRTT

522 performance. (D) Left: Claustrum activity prior to nose poke (average of 0.5 s prior, gray

523 box) was not different between 5CSRTT and 1CSRTT. Paired $t$ test, $t(11)=0.88, P=$

524 0.40. Right: activity immediately subsequent to nose poke (average of $0.5 \mathrm{~s}$

525 subsequent, brown box) was not different between 5CSRTT and 1CSRTT. Paired $t$ test,

$526 t(11)=1.85, P=0.09$. (E) Left: Average claustrum activity aligned to collection of the

527 reinforcement (sucrose pellet) on correct 5CSRTT trials and right: on correct 1CSRTT

528 trials. (F) Left: Claustrum activity prior to collection of reward pellet (average of $0.5 \mathrm{~s}$

529 prior) was greater for the 5CSRTT relative to the 1 CSRTT. Paired $t$ test, $t(11)=3.11, P$

$530=0.01$. Right: activity immediately subsequent to collection (average of $0.5 \mathrm{~s}$

531 subsequent) was greater for the 5CSRTT compared to the 1CSRTT. Paired $t$ test, $t(11)$

$532=4.41, P=0.0010$. 

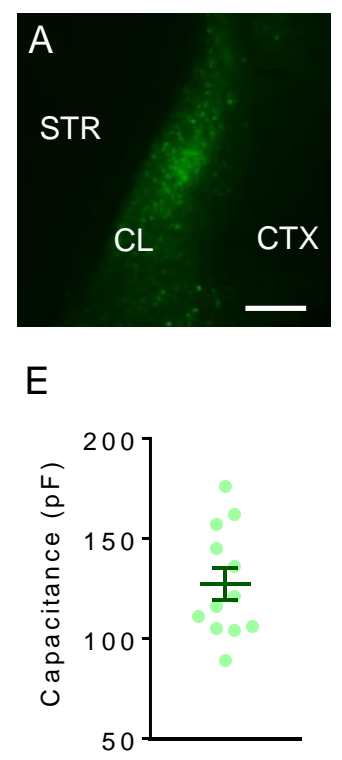
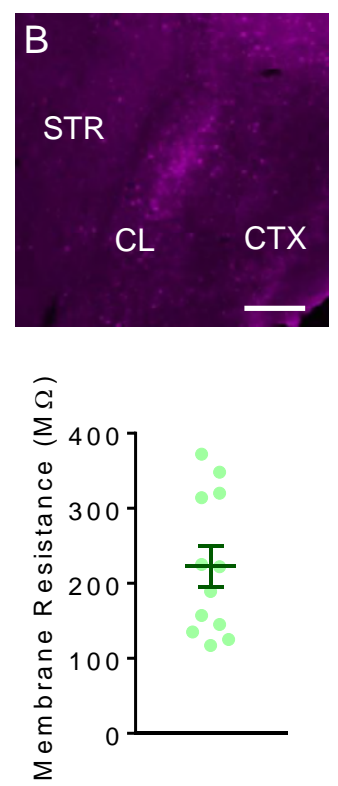
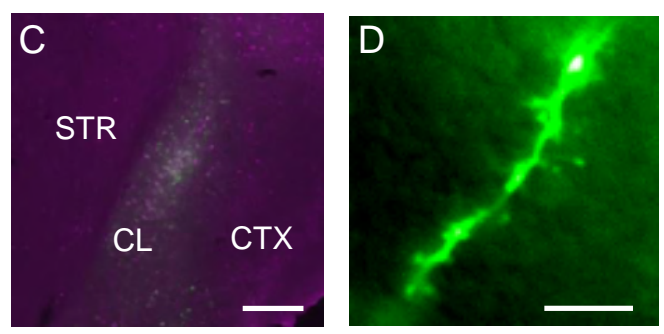

F

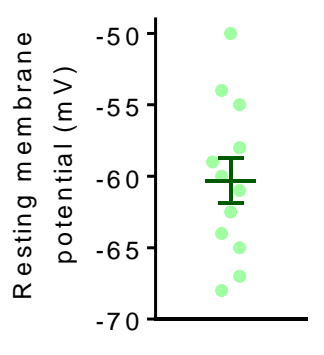

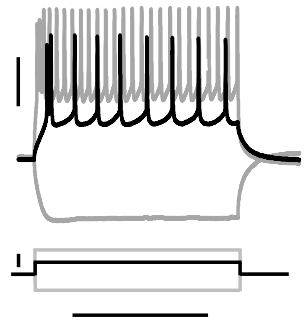

Figure 1 
A

AAV-DIO-eNPhR3.0 or -eYFP

CL

GNB4-cre
B

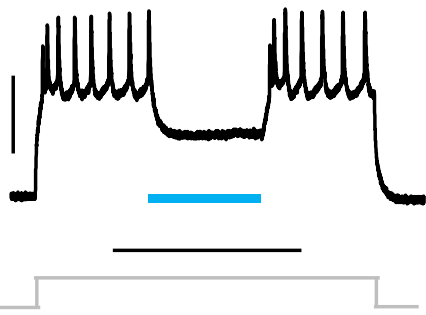

C
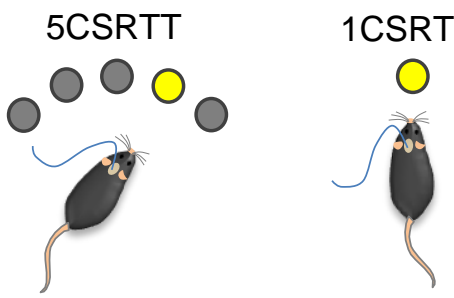

D

$33 \%$ of trials; $470 \mathrm{~nm} 1 \mathrm{~s}$ on; 1 s pre-cue

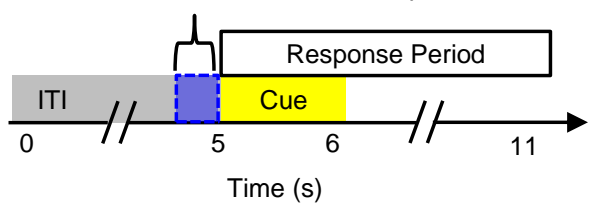

E eNPhR3.0 F

G

$\mathrm{H}$
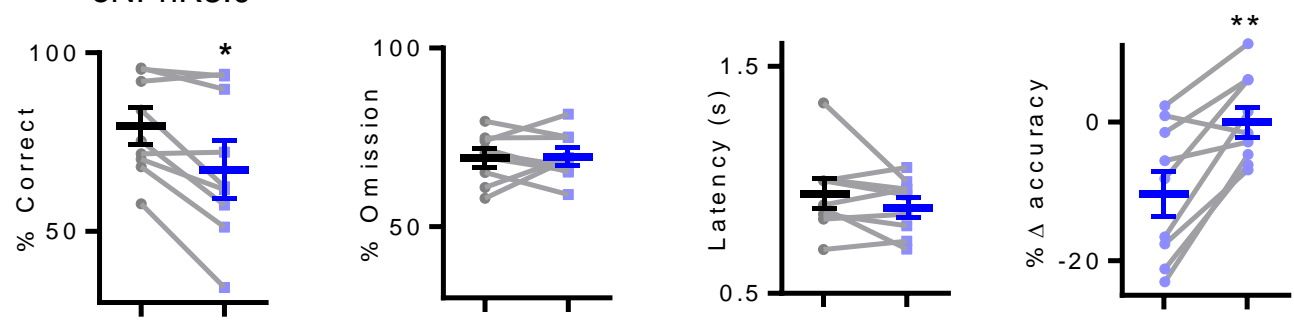

eYFP

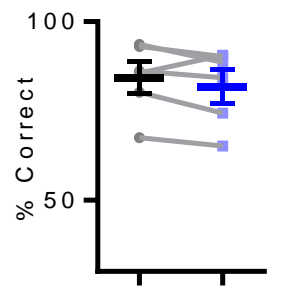

$\operatorname{con} \operatorname{ltrol}_{470} n m$

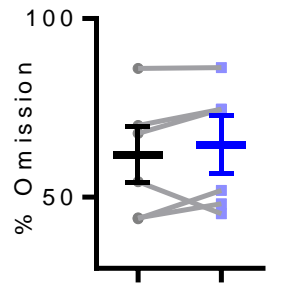

control ${ }_{470} n m$

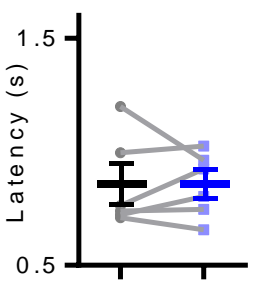

$\operatorname{con}_{470}^{n+0 l} \mathrm{~nm}$

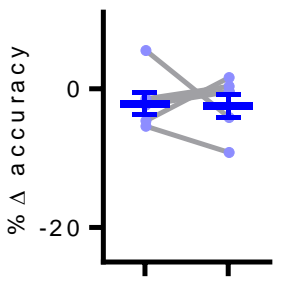

${ }_{5} C^{S^{R T}}{ }_{1}^{T} S^{R T T}$

Figure 2 
A
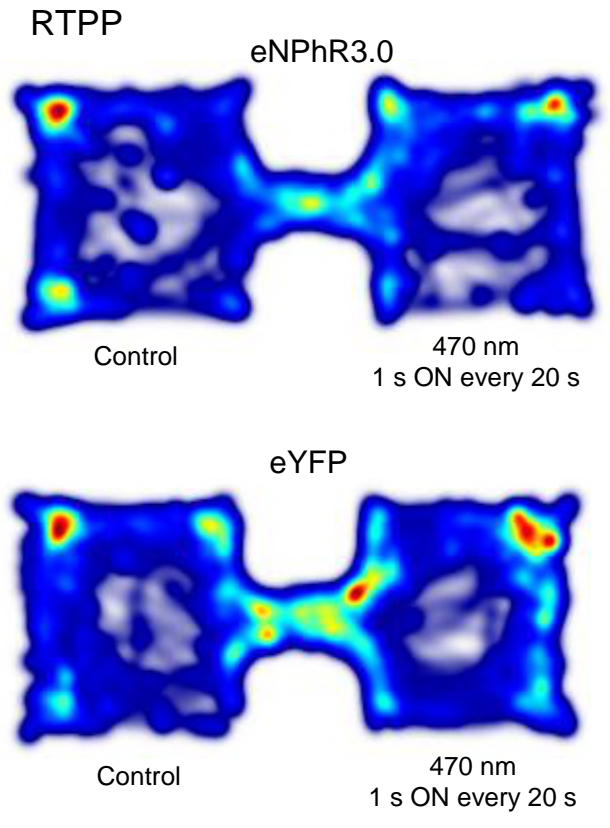

B
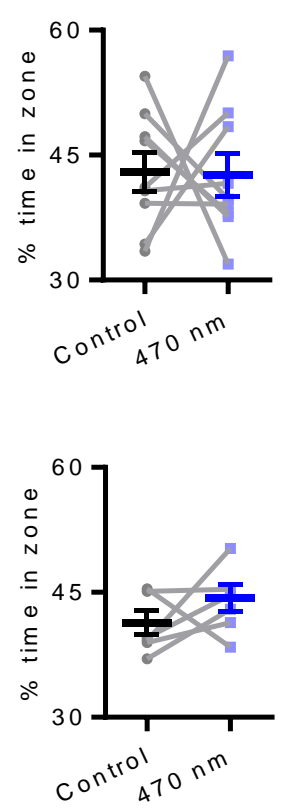

C
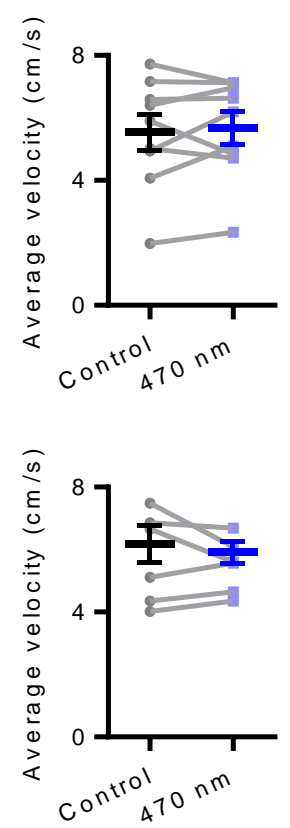

Figure 2 - Supplement 1 
A

Fiber photometry
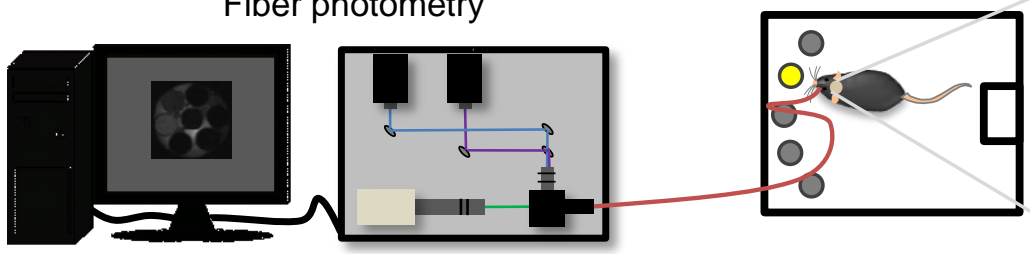

AAV-FLEX-

GCaMP6f Fiber

$\mathrm{CL}$

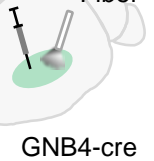

B

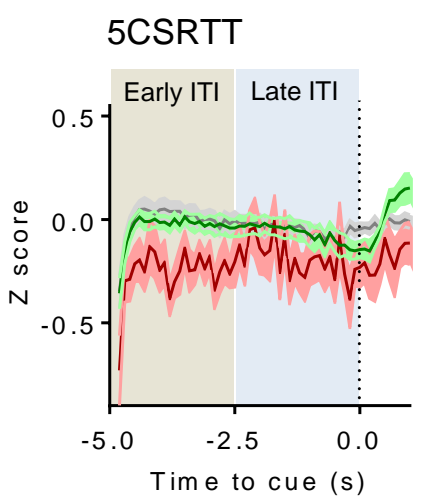

Early ITI

Late ITI

C 5CSRTT

Poke
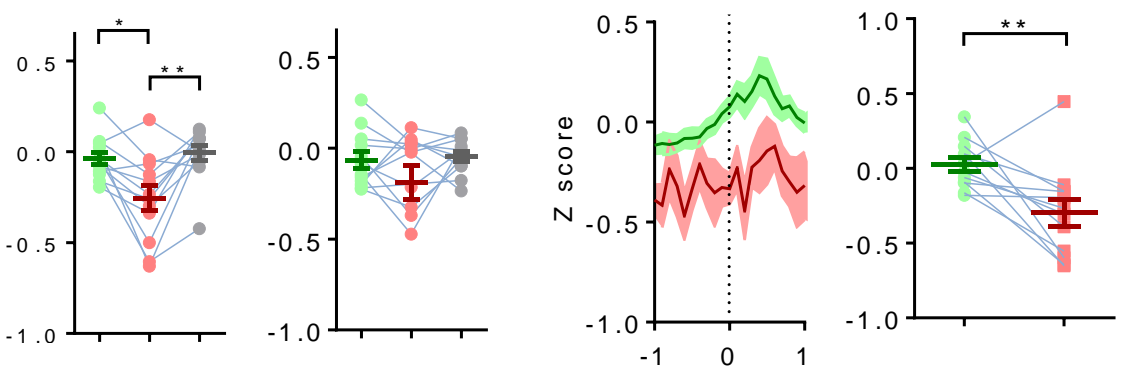

Time to poke (s)

- Correct

- Incorrect

- Om ission

D

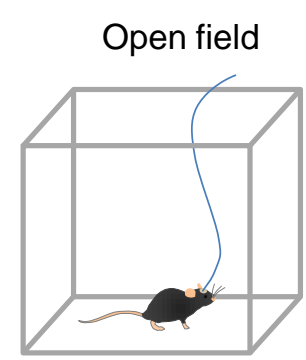

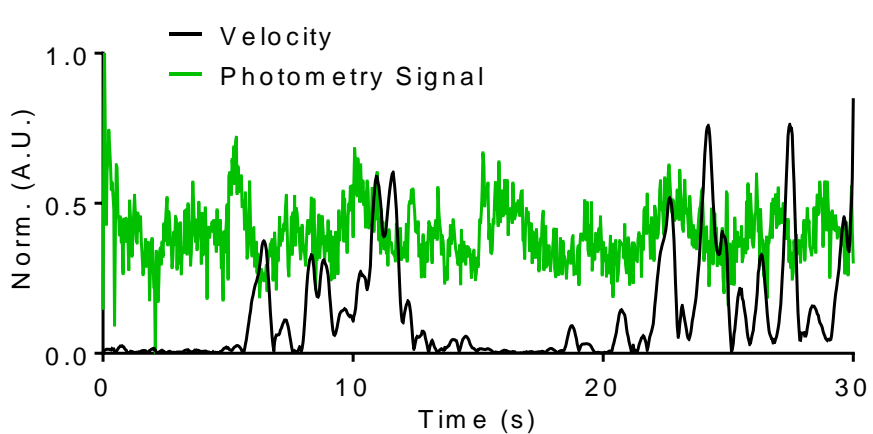

E

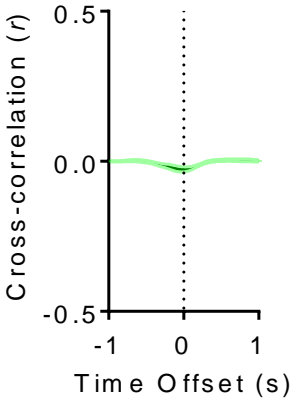

Figure 3 
A

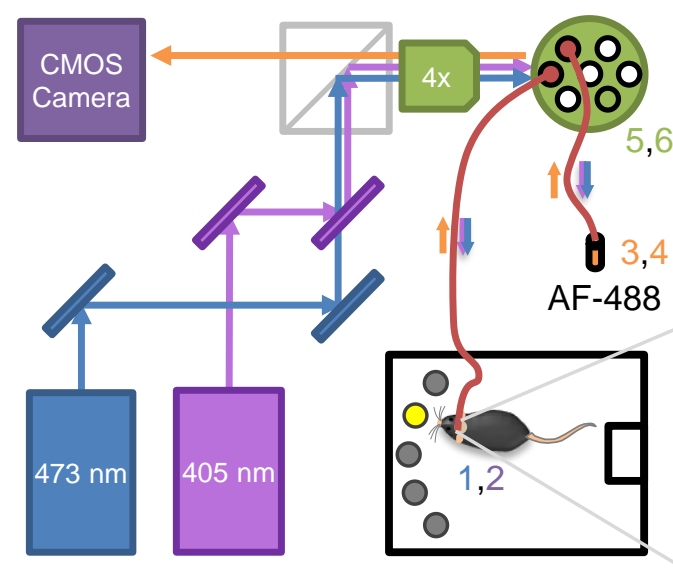

Pulse Sequence

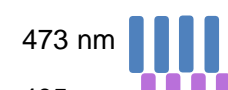

$405 \mathrm{~nm}$

Camera

$$
20 \stackrel{\mathrm{Hz}}{ }
$$

AAV-FLEX-

GCaMP6

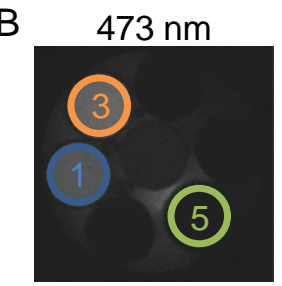

C $\quad$ AF $-488,473 \mathrm{~nm}$

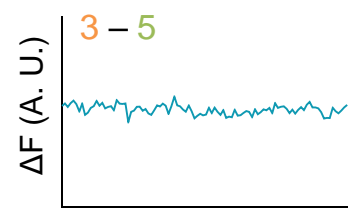

AF- $488,405 \mathrm{~nm}$
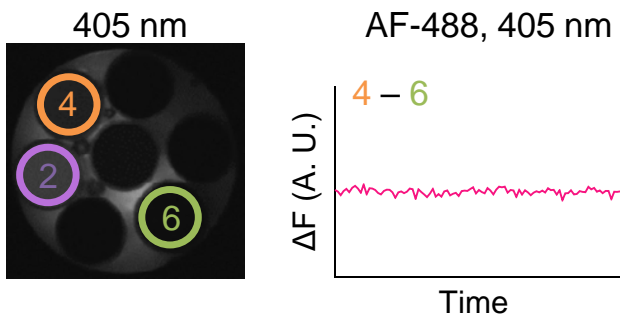

D GCaMP6f signal, $473 \mathrm{~nm}$ excitation

Correct

Incorrect

- Omission

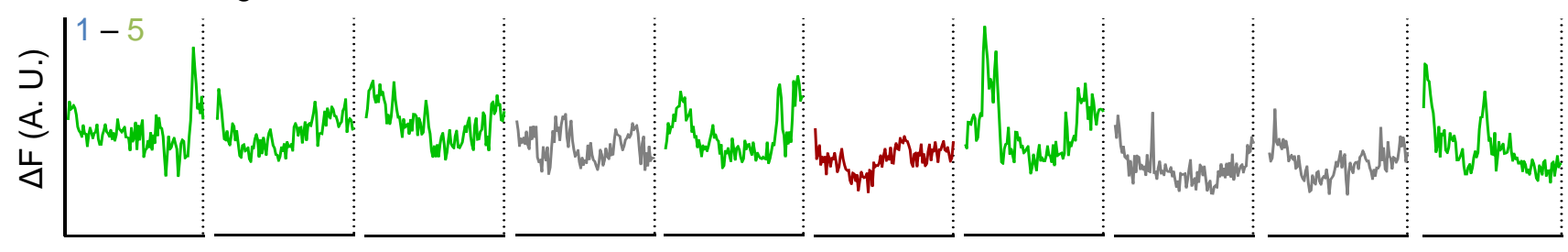

E Autofluorescence signal, $405 \mathrm{~nm}$ excitation

(1)

方

$\mathrm{F}$

Denoised GCaMP6f signal

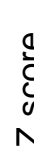

Figure 3 - Supplement 1 
A

Early ITI

Late ITI

B

Early ITI

Late ITI

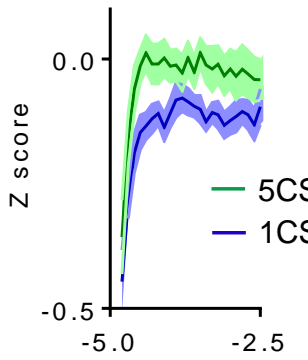

Time to cue (s)

C

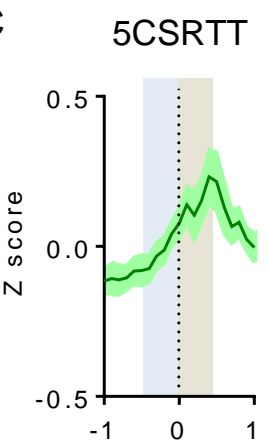

Time to poke (s)

E

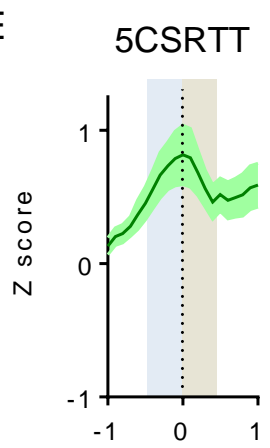

Time to reward (s)

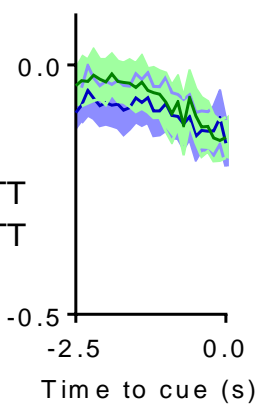

1CSRTT

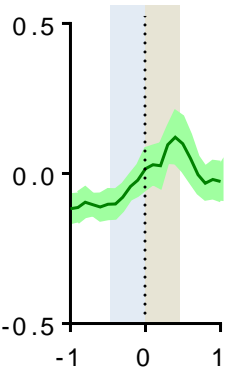

Time to poke (s)
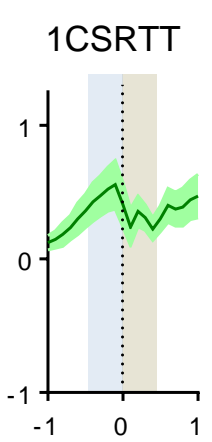

Time to reward (s)

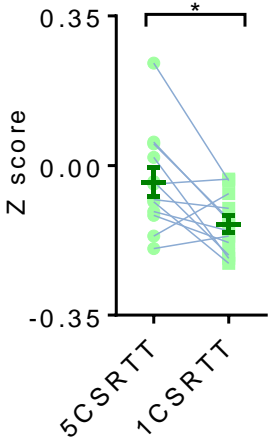

D

Pre-Poke

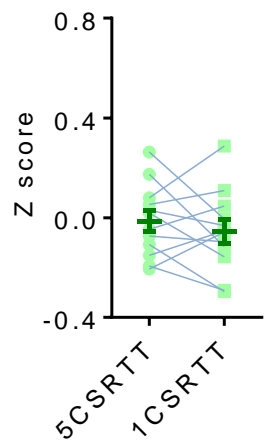

F
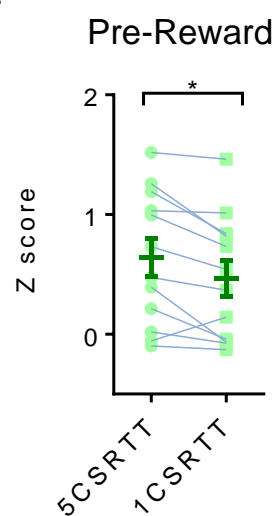
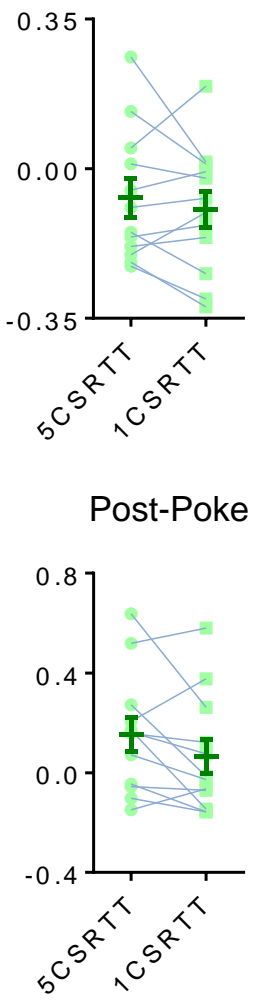

Post-Poke

Post-Reward

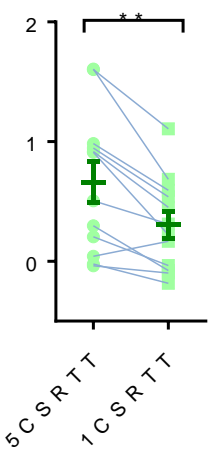

Figure 4 